\title{
EUS-guided Gall Bladder Drainage in Severe Liver Disease: A Single-center Experience in Critically Ill Cirrhotics
}

\author{
Kapil Dev Jamwal*, Manoj Kumar Sharma*, Rakhi Maiwall, Barjesh Kumar Sharma \\ and Shiv Kumar Sarin
}

Department of Hepatology, Institute of Liver and Biliary Sciences, D1, Vasant Kunj, New Delhi, India

\begin{abstract}
Background and Aims: Acute calculous cholecystitis with impending gall bladder perforation in severe liver diseases including decompensated cirrhosis and acute-on-chronic liver failure (ACLF) is difficult to manage, due to the procedures such as cholecystectomy and per cutaneous cholecystostomy being associated with high risk and complications in these patients. Methods: Four cases of severe liver disease with acute calculous cholecystitis who presented to the Institute of Liver and Biliary Sciences (New Delhi, India) for further management were included in the study if they underwent endoscopic ultrasound-guided gall bladder drainage (EUSGBD). The patients were followed up for a minimum of 3 months and outcomes were recorded. Results: Four cases of severe liver disease (three ACLF and one decompensated cirrhosis), with model for end-stage liver disease scores of 24, 26,23 and 25 respectively, presented with acute calculous cholecystitis (Tokyo grade III) and systemic sepsis (high total leukocyte counts). Their international normalized ratios were $2.3,2.6,2.2$ and 2.9 respectively, and two were in shock, requiring inotropes at presentation. Ultrasonography of the abdomen confirmed hugely distended gall bladder with stone impacted at the neck and moderate ascites. All these cases underwent EUS-GBD by linear echo endoscope, and had the gastric wall punctured in the antrum using a 19G access needle followed by dilatation of the tract using controlled radial expansion balloon and Sohendra dilator. In three cases, the plastic stents were placed. In the fourth case, a Nagi stent was placed. All the patients recovered and were discharged within a week. Conclusions: EUS-GBD is challenging in severe liver disease but represents a life-saving procedure, and hence can be attempted in such critically ill patients with utmost care and precaution.
\end{abstract}

Citation of this article: Jamwal KD, Sharma MK, Maiwall R, Sharma BK, Sarin SK. EUS-guided gall bladder drainage in severe liver disease: a single-center experience in critically ill cirrhotics. J Clin Transl Hepatol 2018;6(1):35-39. doi: 10.14218/JCTH.2017.00018.

Keywords: ACLF; Acute-on-chronic liver failure; Cholecystitis; Ascites. Abbreviations: ACLF, acute-on-chronic liver failure; EUS-GBD, endoscopic ultrasound-guided gall bladder drainage.

Received: 10 March 2017; Revised: 1 August 2017; Accepted: 22 August 2017 *Correspondence to: Kapil Dev Jamwal and Manoj Kumar Sharma, Department of Hepatology, Institute of Liver and Biliary Sciences, D1, near Heritage School, Vasant Kunj, New Delhi 110070, India. Tel: +91-11-46300000, Fax: +91-11-4600025, E-mail: drkapil222@gmail.com (KDJ), manojkumardm@gmail.com (MKS)

\section{Introduction}

Acute calculous cholecystitis with impending gall bladder perforation is a medical emergency, requiring emergency treatment (cholecystectomy in medically fit patients). ${ }^{1}$ In patients who are unfit for surgical treatment, percutaneous gall bladder drainage is the second option of treatment. ${ }^{2}$ Endoscopic ultrasound-guided gall bladder drainage (EUSGBD) is an emerging modality of treatment in the group of patients who have acute cholecystitis and are unfit for cholecystectomy, and is currently the treatment of choice in these patients due to their high surgical risks and special situations, such as severe liver disease. ${ }^{3}$

Acute calculous cholecystitis with impending gall bladder perforation in the presence of severe liver disease that includes decompensated cirrhosis and acute-on-chronic liver failure (ACLF) is difficult to manage. This is because of poor coagulation function, abdominal wall collaterals, presence of ascites and high surgical risk of complications (related to both anesthetic and bleeding). In such patients, emergency cholecystectomy has high mortality and morbidity. Yet, percutaneous gallbladder drainage is difficult to perform in these patients, as well, due to presence of ascites and abdominal wall collaterals.

EUS-GBD has been described previously in a randomized controlled trial of patients with acute severe cholecystitis who were unfit for surgery due to poor general condition, but none of these patients were diagnosed with liver disease. ${ }^{3}$ To date, there are no reports of EUS-GBD in patients with severe liver disease.

\section{Methods}

Four cases of severe liver disease with acute calculous cholecystitis who presented to the Institute of Liver and Biliary Sciences (New Delhi, India) for further management were included for study of EUS-GBD in acute cholecystitis and its outcomes. The patients were followed up for a minimum of 3 months. This was a prospective case series study and the EUS-GBD was applied as a life-saving maneuver.

Inclusion criteria were age $>18$ years, classification as unfit for cholecystectomy (American Society of Anesthesiologist score: IV), blocked cystic duct due to a stone (as seen on imaging), failed transpapillary drainage attempt, and diagnosis of severe liver disease (ACLF or decompensated chronic liver disease). Exclusion criteria were failure to obtain valid consent, classification as unfit for anesthesia, or evidence of any of the following: diffuse mucosal bleeding or 
uncorrectable coagulopathy (after correction of thromboelastograph parameters); perivesicular fluid (since chances of leak and complications will increase as the walls may not oppose) or floating gall bladder that was distant $(>2 \mathrm{~cm}$ ) from the stomach or duodenal wall; ectopic gastric varices (isolated gastric varices type 2) and/or duodenal varices; altered stomach anatomy or proximal duodenal anatomy, such as post-gastrectomy status or a gastric outlet obstruction.

Instruments used for the procedure included a linear echo endoscope (UCT 180; Olympus Japan), EU-ME 1 processor (Olympus), controlled radial expansion balloon (Boston Scientific, USA), Visiglide exchange guidewire (0.032; Olympus), endoscopic ultrasound access needle 19G (Cook Medical, USA), Sohendra biliary dilator (Olympus), Nagi selfexpanding metallic stent (Taewong Co., Korea), and doublepigtail plastic stents $(7 \mathrm{Fr} \times 4 \mathrm{~cm}$ and $10 \mathrm{Fr} \times 4 \mathrm{~cm}$; Indomed Co., India). The procedures were carried out with the patient in left lateral position and under fluoroscopy guidance. The sedation used was propofol, which was administered by a senior anesthetist. No patient was placed on mechanical ventilation for the procedure.

\section{Results}

Four patients with severe liver disease (three with ACLF and one with decompensated cirrhosis) having model of endstage liver disease scores of 24, 26, 23 and 25 respectively presented with acute calculous cholecystitis (Tokyo grade III) ${ }^{4}$ and systemic sepsis (total leukocyte counts of 20430, 23543, 17986 and $21121 \mathrm{~mm}^{3}$ respectively). Their international normalized ratios were 2.3, 2.6, 2.2 and 2.9 respectively, and two presented with shock requiring inotropes (Table 1). All four patients were transfused with fresh frozen plasma, platelet rich concentrates (PRCs) and cryoprecipitates prior to the procedure, depending upon thromboelastograph findings (Table 2).

Thromboelastograph measures the dynamic viscoelastic property of blood during coagulation, and its use has been shown to decrease the usage of blood products as compared to conventional parameters such as platelets, international normalized ratio, bleeding time or clotting time. The thromboelastograph has been used primarily for trauma surgery and for surgeries involving liver resection or transplantation. We use thromboelastograph in our center for all severe liver disease patients, for both surgical and endoscopic procedures. $^{5}$

For all four patients, abdominal ultrasound showed gall bladder stones, hugely distended gall bladder with moderate ascites (median wall thickness of gall bladder was $6.2 \mathrm{~mm}$, as measured by abdominal ultrasound or endoscopic ultrasound) and abdominal wall collaterals. Abdominal computed tomography (Fig. 1) and magnetic resonance cholangiopancreatography (Fig. 2) was performed in two patients and showed ascites with hugely distended gall bladder, impacted stone in cystic duct (magnetic resonance cholangiopancreatography; Fig. 2), abdominal collaterals (intra-abdominal and abdominal wall) and perivesicular fluid. All four patients were deemed high risk for percutaneous drainage due to the presence of ascites, coagulopathy, high model for end-stage liver disease score and abdominal wall collaterals. Hence, after discussion with each of the patients and their representatives, EUS-GBD was agreed to and performed.

For all four patients, the gastroscopy prior to the endoscopic ultrasound showed varices (ranging from small to large). The linear array echo endoscope was applied and showed the presence of ascites in the perihepatic space; an avascular site was also identified from the antrum (in all the patients the gall bladder access was from the gastric antrum). The gastric wall was punctured with the 19G access needle and the fundus of the gall bladder was punctured, after which an exchange guidewire (0.0032) was passed into the gall bladder lumen and coiled (Fig. 3). This was followed by dilatation of the tract using a controlled radio expansion balloon dilator (Fig. 4) up to $8 \mathrm{~mm}$ in two patients, and with Sohendra biliary dilators up to $10 \mathrm{~F}$ in the other two patients.

The cholecystogram was avoided anticipating any complications (i.e. perforation due to increased intra-gall bladder pressure or leak at puncture site, as plastic stents were planned for deployment) after instillation of contrast into the gall bladder. After the puncture, purulent material was aspirated in all the cases, confirming the presence of pus. In three

Table 1. Patient characteristics

\begin{tabular}{|c|c|c|c|c|}
\hline Characteristic & Patient 1 & Patient 2 & Patient 3 & Patient 4 \\
\hline Age, years & 34 & 56 & 39 & 54 \\
\hline Diagnosis & ACLF & Decompensated cirrhosis & ACLF & ACLF \\
\hline Hemoglobin, gm/dL & 8.2 & 7.9 & 9.1 & 8.5 \\
\hline TLC, $\mathrm{mm}^{3}$ & 20430 & 23543 & 17986 & 21121 \\
\hline Platelet, $1000 / \mathrm{mm}^{3}$ & 54 & 75 & 67 & 59 \\
\hline Bilirubin, mg/dL & 21.3 & 8.0 & 15.1 & 11.8 \\
\hline International normalized ratio & 2.3 & 2.6 & 2.9 & 2.2 \\
\hline Model for end-stage liver disease score & 24 & 26 & 23 & 25 \\
\hline Tokyo grade & III & III & III & III \\
\hline Inotropes, noradrenaline, $\mu \mathrm{g} / \mathrm{min}$ & Yes (5) & No & Yes (3) & No \\
\hline Collaterals on percutaneous abdominal ultrasound & Yes & No & Yes & Yes \\
\hline Ascites grade & Yes (3) & Yes (2) & Yes (3) & Yes (3) \\
\hline
\end{tabular}

All of the patients are separate and the patient with the stent exchange has not been included in this table. 
Jamwal K.D. et al: EUS gall bladder drainage in liver disease

Table 2. Patient characteristics related to the procedure

\begin{tabular}{|c|c|c|c|c|}
\hline Characteristics & Patient 1 & Patient 2 & Patient 3 & Patient 4 \\
\hline Stent placed & Nagi (SEMS) & Plastic & Plastic & Plastic \\
\hline $\begin{array}{l}\text { Products transfused (FFP's/PRC's/Cryo) } \\
\text { based on thromboelastograph }\end{array}$ & Yes $(3 / 3 / 0)$ & Yes $(3 / 0 / 6)$ & Yes $(3 / 0 / 0)$ & Yes $(3 / 3 / 0)$ \\
\hline Complication & $\begin{array}{l}\text { Mild pain and } \\
\text { migrated stent }\end{array}$ & None & None & None \\
\hline Procedure duration in min & 96 & 56 & 52 & 50 \\
\hline Outcome & $\begin{array}{l}\text { Discharged } \\
\text { at } 5 \text { days }\end{array}$ & $\begin{array}{l}\text { Discharged } \\
3 \text { days }\end{array}$ & Discharged 5 days & $\begin{array}{l}\text { Discharged } \\
7 \text { days }\end{array}$ \\
\hline Bilirubin at 3 months, $\mathrm{mg} / \mathrm{dL}$ & 2.9 & 1.7 & 3.2 & 1.5 \\
\hline Mean follow-up in days & 327 & 117 & $\begin{array}{l}\text { Underwent liver transplant } \\
\text { after } 90 \text { days }\end{array}$ & 194 \\
\hline
\end{tabular}

Abbreviations: Cryo, cryoprecipitates; FFP, fresh frozen plasma; PRC, platelet rich concentrates; SEMS, self-extending metallic stent.

of the patients, plastic stents (double-pigtail $10 \mathrm{Fr} \times 4 \mathrm{~cm}$ in two of the patients, and $7 \mathrm{~F} \times 4 \mathrm{~cm}$ in one) (Fig. 5) were deployed, and in one case, a Nagi stent $(10 \mathrm{~mm} \times 5 \mathrm{~cm})$ was deployed (Fig. 6).

The technical and clinical success rates were $100 \%$ and there were no immediate complications in the form of puncture site bleeding, hematoma, bile leak or spontaneous bacterial peritonitis noted. Day 2 post-procedure imaging (abdominal ultrasound; Fig. 7) confirmed presence of stents in situ and decompressed gall bladder. All the patients recovered satisfactorily and were discharged within a week. There was no major long term complication reported; however, one patient complained of mild pain after the stent placement and that same patient experienced migration of the self-expanding metallic stent after 3 months. One patient required replacement of the plastic stent at 3 months.

All the patients are being closely followed up, with a minimum follow-up period of 6 months and maximum of 1 year. One patient underwent successful liver transplant after 3 months. We noted a steady decrease in the serum bilirubin level and in the liver disease status as the coagulopathy improved after the recovery from sepsis (Table 2 ).

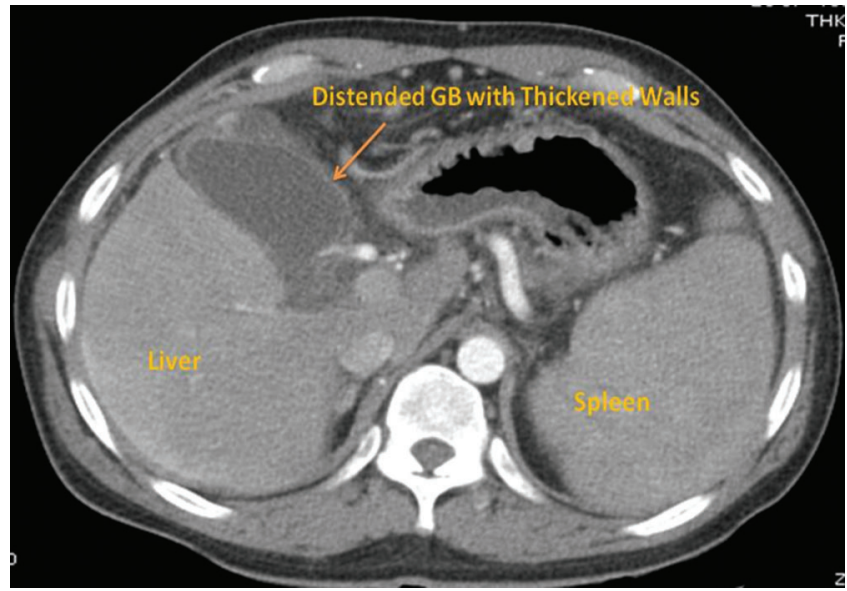

Fig. 1. Abdominal computed tomography sagittal section in the arterial phase, made 2 days prior to drainage and showing thickened gall bladder and shrunken liver.

\section{Discussion}

EUS-GBD is comparable to percutaneous drainage in terms of decreased complications and lower rates of re-intervention, catheter migration and repeat procedures in cases where cholecystectomy is contraindicated. ${ }^{6}$ Technically, the new procedure is more challenging in terms of the gall bladder localization (floating, fixed to undersurface of liver or adhesions) and its status as a mobile organ. The other concern is the thickness of the gall bladder wall, which is thin compared to a bile duct or pancreatic fluid collections, so any forceful manipulation on the wall opposite of the puncture could lead to wall rupture or trauma.

EUS-GBD has concerns of bile leakage from the puncture site, pneumoperitoneum, gall bladder perforation due to increased manipulation inside the gall bladder lumen and stent migration. Also, there is concern of availability of technical expertise for this procedure, as there is a high learning curve and referral bias. However, a recent multicentric trial by Walter et al. ${ }^{7}$ using lumen-opposing metal stents has shown it to be very efficacious and safe. There is

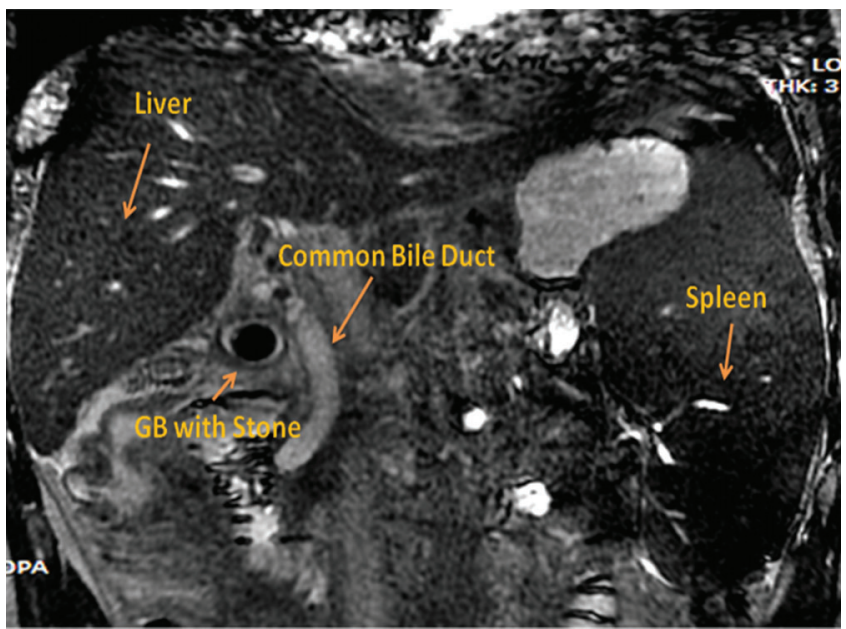

Fig. 2. Magnetic resonance cholangiopancreatography coronal view, made 1 day prior to drainage and showing an impacted stone in the cystic duct. 


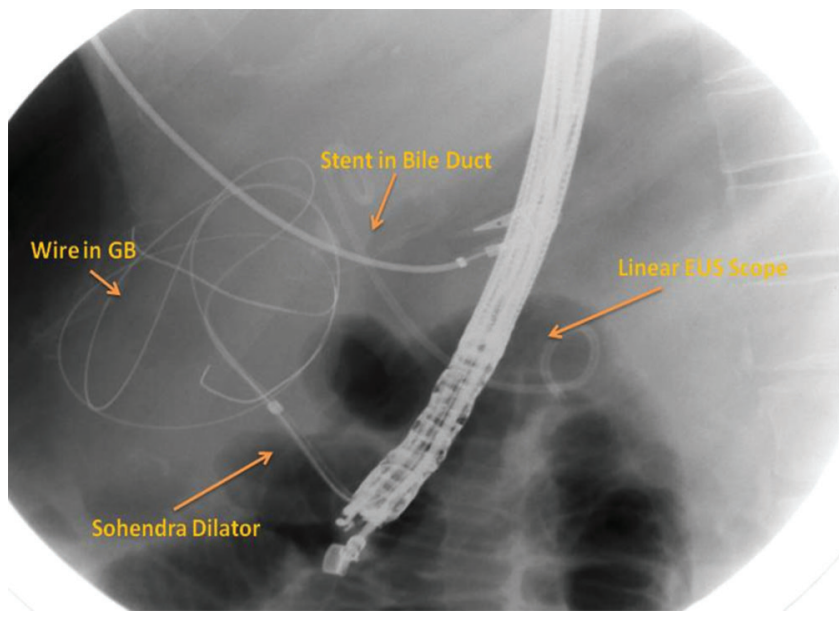

Fig. 3. Fluoroscopy image made during the procedure and showing dilatation of the tract achieved by a Sohendra biliary dilator over a radio opaque guidewire coiled in gall bladder and a stent in the bile duct.

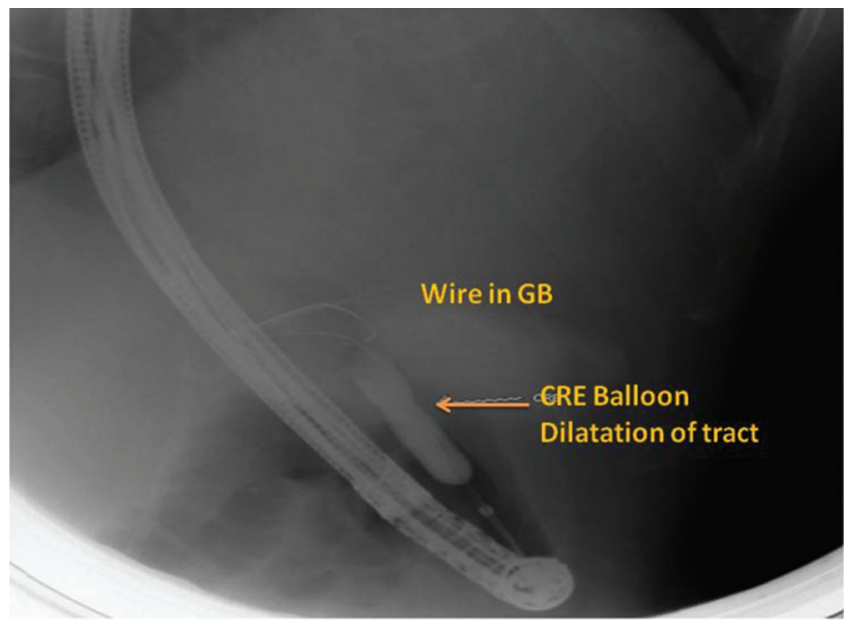

Fig. 4. Fluoroscopy image made during the procedure and showing the dilatation of the tract using a controlled radio expansion balloon over a radio opaque guidewire placed in the gall bladder.

no literature available as of yet on EUS-GBD in liver disease patients. The usual indications of percutaneous gall bladder drainage are advanced age, unfit for surgery status, advanced malignancies, failed medical therapy, lower bile duct obstruction with patent upper bile duct and hepatic duct, and poor surgical candidate status. ${ }^{6}$

The other problem which may arise in these patients with transmural gall bladder drainage is the stent block and subsequent exchange. The multicentric trial published by Walter et $a .^{7}$ also showed that the stent can be placed for a long time ( 3 months). In our study, one patient required stent exchange, while the other two patients did well and no stent exchange was required. This finding signifies the possibility of a spontaneous fistula formation between gall bladder and the gastric wall leading to spontaneous decompression of the gall bladder and, hence, no recurrence of symptoms. We also noted a decreased procedure time after the first procedure, although the number is too low to make further recommendations.

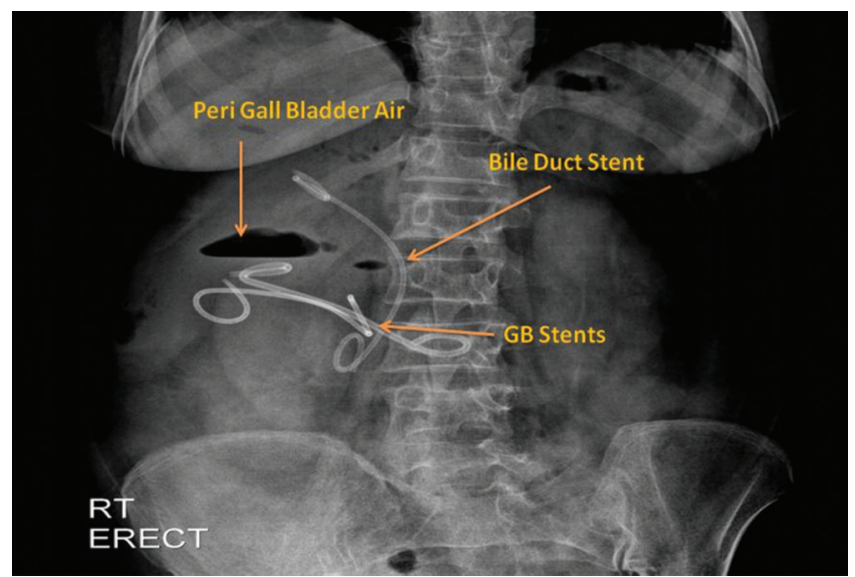

Fig. 5. Abdominal X-ray showing two plastic stents (double-pigtail) in the gall bladder and a plastic double-pigtail stent in the bile duct, made 2 days post-procedure. There is evidence of pneumoperitoneum, which resolved spontaneously.

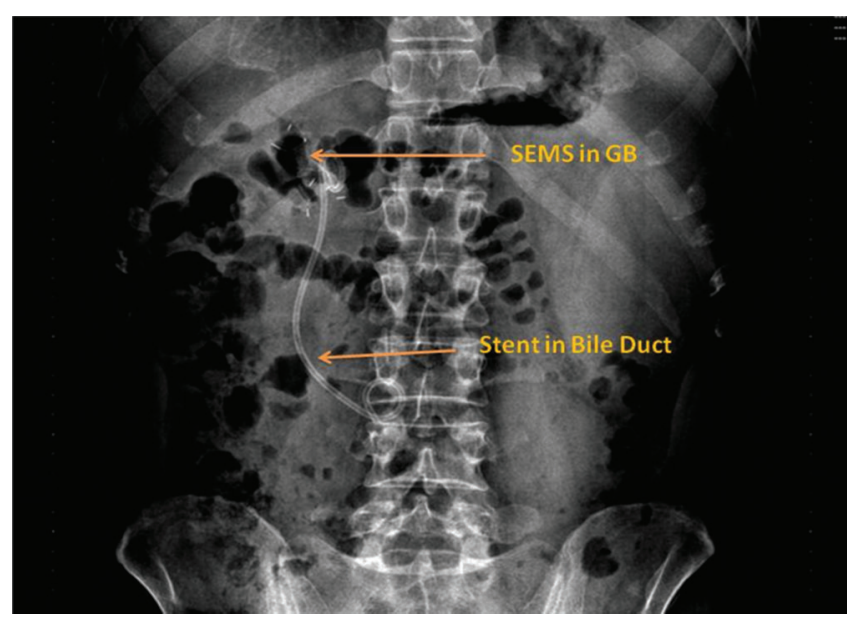

Fig. 6. Abdominal $X$-ray showing a self-expanding metal stent (Nagi stent) in the gall bladder and a plastic double-pigtail stent in the bile duct, made 2 days post-procedure. There is no evidence of pneumoperitoneum.

EUS-GBD is a safe procedure, but there are still unanswered questions related to its safety, such as the optimal site of puncture and stent deployment (gastric or duodenal wall), duration of stent placement, timing of exchange, whether the lumen-opposing metal stents which has been developed for pancreatic fluid collections will be a good indication for EUS$\mathrm{GBD}$, and whether the present accessories are correct or will specialized accessories be required for this procedure (e.g. a shorter exchange wire or smaller controlled radio expansion dilator in length). ${ }^{7,8}$ Although we did not encounter any major complications, complications do occur. Hence, these procedures should be done with utmost care and all precautions to avoid complications should be taken beforehand.

The long-term outcomes of EUS-GBD are not available, but there are concerns of formation of post-procedure adhesions between the gastric wall or the duodenal wall, which may lead to difficult cholecystectomy or of any surgical procedure if required in the future of these patients. Hence, this procedure should be done according to very select indications. 


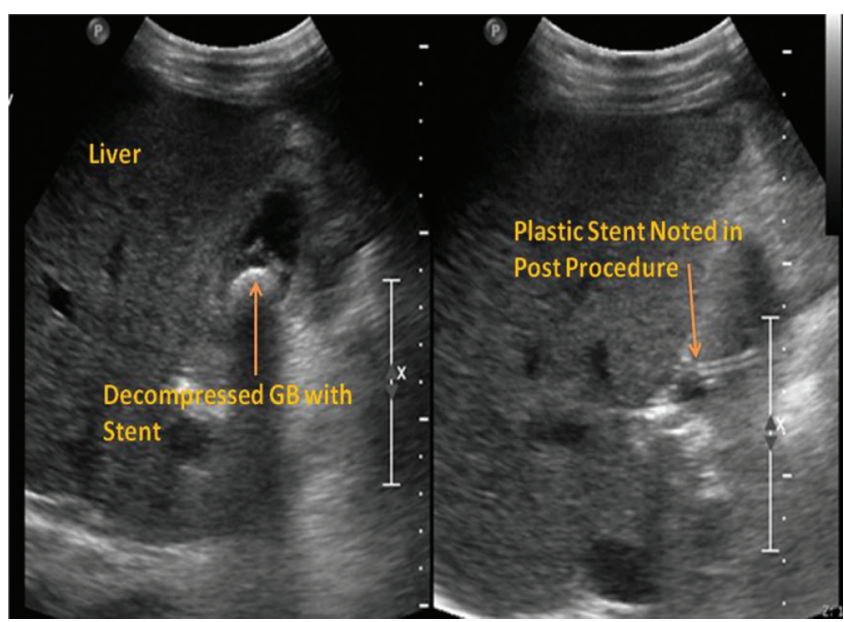

Fig. 7. Abdominal ultrasound made 2 days post-procedure shows a decompressed gall bladder with stent inside (Left) and the plastic stent entering into the gall bladder (Right).

All the procedures in this case series were performed by a highly-skilled endoscopist, with more than 1000 endoscopic retrograde cholangiopancreatography and endoscopic ultrasound procedures as well as experience of more than 200 therapeutic endoscopic ultrasound procedures. The limitations of our study include small numbers of patients, no comparison to the standard treatment (percutaneous cholecystostomy) and the long-term outcome available for only one of the four patients assessed.

\section{Conclusions}

Endoscopic ultrasound-guided biliary procedures in severe liver disease are challenging but life-saving, and hence expanding the role of endoscopic ultrasound in these patients should be explored. EUS-GBD is one of these difficult procedures and can be attempted in patients with severe liver disease and acute severe cholecystitis who are at a higher risk of complications during cholecystectomy or percutaneous drainage.

\section{Conflict of interest}

The authors have no conflict of interests related to this publication.

\section{Author contributions}

Performed the procedure, made the management plan, and performed the follow up (KDJ), gave clinical input, edited the report and participated in the follow up (MKS, RM, BKS, SKS).

\section{References}

[1] Wilson RG, Macintyre IM, Nixon SJ, Saunders JH, Varma JS, King PM. Laparoscopic cholecystectomy as a safe and effective treatment for severe acute cholecystitis. BMJ 1992;305:394-396.

[2] Akyürek N, Salman B, Yüksel O, Tezcaner T, Irkörücü O, Yücel C, et al. Management of acute calculous cholecystitis in high-risk patients: percutaneous cholecystotomy followed by early laparoscopic cholecystectomy. Surg Laparosc Endosc Percutan Tech 2005;15:315-320.

[3] Jang JW, Lee SS, Song TJ, Hyun YS, Park DY, Seo DW, et al. Endoscopic ultrasound-guided transmural and percutaneous transhepatic gallbladder drainage are comparable for acute cholecystitis. Gastroenterology 2012; 142:805-811. doi: 10.1053/j.gastro.2011.12.051.

[4] Yokoe M, Takada T, Strasberg SM, Solomkin JS, Mayumi T, Gomi H, et al. New diagnostic criteria and severity assessment of acute cholecystitis in revised Tokyo Guidelines. J Hepatobiliary Pancreat Sci 2012;19:578-585. doi: 10. 1007/s00534-012-0548-0.

[5] Theusinger OM, Wanner GA, Emmert MY, Billeter A, Eismon J, Seifert B, et al. Hyperfibrinolysis diagnosed by rotational thromboelastometry (ROTEM) is associated with higher mortality in patients with severe trauma. Anesth Analg 2011;113:1003-1012. doi: 10.1213/ANE.0b013e31822e183f.

[6] Baron TH, Grimm IS, Swanstrom LL. Interventional approaches to gallbladder disease. N Engl J Med 2015;373:357-365. doi: 10.1056/NEJMra1411372.

[7] Walter D, Teoh AY, Itoi T, Pérez-Miranda M, Larghi A, Sanchez-Yague A, et al. EUS-guided gall bladder drainage with a lumen-apposing metal stent: a prospective long-term evaluation. Gut 2016;65:6-8. doi: 10.1136/gutjnl-2015309925.

[8] Moon JH, Choi HJ, Kim DC, Lee YN, Kim HK, Jeong SA, et al. A newly designed fully covered metal stent for lumen apposition in EUS-guided drainage and access: a feasibility study (with videos). Gastrointest Endosc 2014;79:990995. doi: $10.1016 /$ j.gie.2014.02.015. 